\title{
Isotope effects and molecular orbital theory
}

\section{同位体効果と分子軌道理論}

\author{
Takao OI \\ Department of Chemistry, Sophia University, 7-1 Kioicho, Chiyodaku, Tokyo 102-8554, Japan \\ Tel: +81-3-3238-3359 Fax:+81-3-3238-3361 e-mail:t-ooi@sophia.ac.jp
}

( Received 29, September $2004 \quad$ Accepted 25, November 2004 )

A brief explanation of isotope effects that are quantum mechanical effects and their theoretical foundation are given. Application of molecular orbital calculations to the elucidation of isotope effects is briefly reviewed with two examples.

Keyword: Isotopes, Isotope effects, Quantum mechanics, Molecular orbital calculations, Reduced partition function ratios

\section{INTRODUCTION}

Natural water is a mixture of light and heavy water. If the deuterium (heavy hydrogen)-toprotium (light hydrogen) ratio in liquid water is compared with that in vapor in equilibrium with the liquid, the liquid is found to be richer in deuterium by a factor of about 1.07 than the vapor at room temperature. If water is electrolyzed, oxygen gas appears at the anode and hydrogen gas at the cathode. A comparison of the deuterium abundance in the water being electrolyzed with that in the hydrogen gas evolved shows that the water is again richer in deuterium, but this time by a factor of 4 to 9 . The exact difference depends on the conditions of electrolysis including the electrode materials. These two phenomena illustrate isotope effects; isotopomers, chemical species that are different from each other in isotopic compositions and the molecular structural positions, behave physically and chemically slightly differently.

Isotope effects in chemical exchange reactions and the corresponding isotope fractionation are quantum mechanical effect and cannot be explained by the classical statistical mechanics. When isotopic atoms are distributed between two chemical species, the preference of an isotope for associating itself with one chemical species rather than the other is determined by the chemical environments provided by the two species competing for the isotope in concern. The structure and molecular forces can, therefore, be elucidated through examination of the distribution of the isotopes between two chemical species, which is the basis of the science of isotope effects and is in contrast to the methodology used in isotopic tracer studies.

In this review, basic theory of equilibrium isotope effects and calculations of isotope effects based on the molecular orbital theory are presented.

\section{ISOTOPE EXCHANGE EQUILIBRIA AND ISOTOPIC REDUCED PARTITION FUNCTION RATIOS}

Isotope effects based on molecular translational, rotational and vibrational motions can be theoretically estimated by calculating isotopic reduced partition function ratios (rpfrs) of the chemical species participating in the isotope exchange reaction in concern. That reaction may be expressed, without losing any generality, as 
$\mathrm{AX}+\mathrm{BX}^{\prime}=\mathrm{AX}^{\prime}+\mathrm{BX}$

where $X$ and $X^{\prime}$ are the heavier and lighter isotopes of the element in concern and $\mathrm{A}$ and $\mathrm{B}$ are polyatomic groups. The equilibrium constant, $K$, of Reaction (1) (strictly speaking, the equilibrium constant estimated quantum mechanically divided by that estimated classically) can be given as

$\ln K=\ln \left(s / s^{\prime}\right) f_{\mathrm{BX}}-\ln \left(s / s^{\prime}\right) f_{\mathrm{AX}}$,

where $\left(s / s^{\prime}\right) f_{\mathrm{AX}}$ and $\ln \left(s / s^{\prime}\right) f_{\mathrm{BX}}$ are the rpfrs of the chemical species AX and BX, respectively. The general formula of the rpfr of a species is given, under the Born-Oppenheimer and harmonic oscillator approximations, as ${ }^{1}$

$\left(s / s^{\prime}\right) f=\prod_{i=1}^{l} \frac{u_{i} \exp \left(-u_{i} / 2\right) /\left\{1-\exp \left(-u_{i}\right)\right\}}{u_{i}^{\prime} \exp \left(-u_{i}^{\prime} / 2\right) /\left\{1-\exp \left(-u_{i}^{\prime}\right)\right\}}$

where

$u_{i}=h c \omega_{i} /(k T)$,

and

$u_{i}^{\prime}=h c \omega^{\prime}{ }_{i} /(k T)$.

Here, $l$ is the degree of freedom of the vibrational motion, $h$ the Planck's constant, $c$ the velocity of light, $\omega_{i}$ and $\omega^{\prime}{ }_{i}$ are the wavenumbers of the $i$ th molecular vibration of the heavier and lighter isotopomers, respectively, $k$ the Boltzmann's constant and $T$ the absolute temperature. Eq. (3) means that one can calculate the rpfr of a chemical species at a given temperature if all the vibrational frequencies of the isotopic species in concern are known.

A full experimental determination of all the isotopic frequencies would be the ideal way of using Eq. (3). Except for very simple molecules, however, this is still impossible at the present level of science due mainly to experimental difficulties; vibrational spectra do not reveal all fundamental frequencies and those obtained for solutions and solids integrate the effects from numerous molecular environments that cannot be addressed individually. Since a small error in the measurement of isotopic frequencies leads to a large error in the corresponding rpfr, it would be more productive to calculate theoretical vibrational frequencies and to use them in model calculations of isotope effects.

For well-defined molecules, it is a common practice to calculate vibrational frequencies by the vibrational analysis using, for example, Wilson's $\boldsymbol{G F}$ matrix method. In this analysis, the force fields that best reproduce the observed frequencies are first constructed, and then all the frequencies of the isotopomers are calculated using the best force field thus obtained. For chemical species in condensed phases, such a model like cell model ${ }^{2}$ has often been utilized which seems to oversimplify the treatment of the condensed phases.

\section{MOLECULAR ORBITAL CALCULATIONS OF REDUCED PARTITION FUNCTION RATIOS}

With the rapid progress in computer technology, it is now possible to carry out $a b$ initio and other molecular orbital (MO) calculations of vibrational frequencies for rather large and complicated chemical species and those in the condensed phase. Various program packages are available for computational quantum chemistry. The Gaussian series ${ }^{3}$ is an example. The procedure for the estimation of rpfrs and corresponding equilibrium constants of isotope exchange reactions is shown as a flowchart in Fig.1 and is briefly as follows: First, one constructs a model chemical species. For example, if one would like to calculate the rpfr of a lithium ion in aqueous solution, one assumes hydrated lithium ions, $\mathrm{Li}^{+}\left(\mathrm{H}_{2} \mathrm{O}\right)_{n}$, with $n$ the hydration number as the lithium ion in aqueous solution. The structure of the 
$\mathrm{Li}^{+}\left(\mathrm{H}_{2} \mathrm{O}\right)_{n}$ species is then optimized, that is, the geometries at global or local minima of the potential energy surfaces are obtained, and vibrational frequencies are calculated at the optimized geometries. Frequencies are also calculated for isotope substituted species.

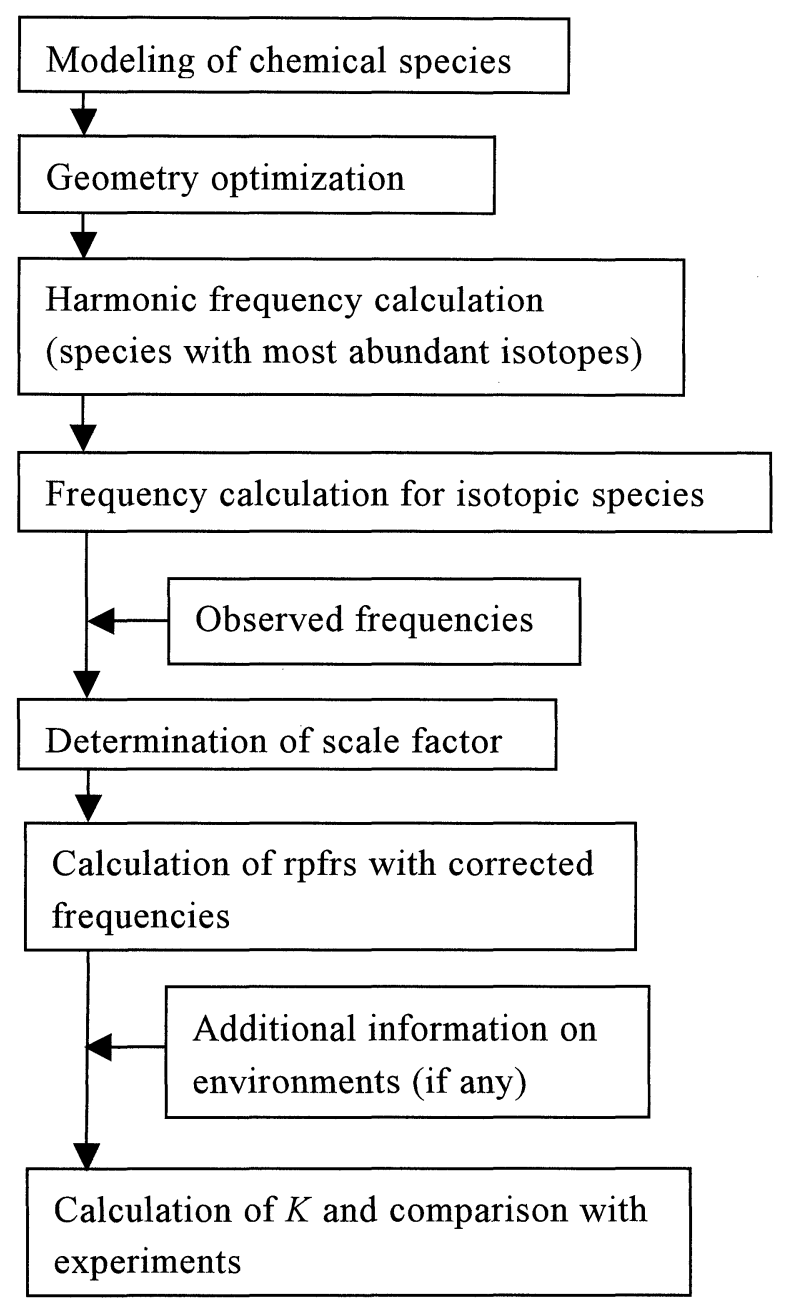

Fig.1. The procedure for the calculations of the reduced partition function ratios and corresponding equilibrium constant of isotope exchange reaction.

If experimental frequencies are available, the scaling factor is calculated for scaling frequencies by comparing the observed and calculated frequencies, because calculated frequencies often do not agree with observed ones. When experiment is not available, an empirical scale factor may be used. Using the frequencies of the isotopic pair of the chemical species corrected by the scale factor, one can calculated the rpfr of the species with eq. (3), and after calculating the rpfr of the counterpart species, one can calculate the equilibrium constant of the isotope exchange reaction (1) with eq. (2).

\section{EXAMPLES OF MOLECULAR ORBITAL CALCULATIONS
IV-A. Lithium isotope effects in aqueous ion exchange systems

A variety of experiments are reported on lithium isotope separation by ion exchange chromatography. ${ }^{4}$ The isotope separation here is based on the equilibrium lithium isotope effects between lithium ions in the aqueous solution phase and those in the ion exchange resin phase, and the equilibrium constant of this reaction is experimentally obtained and is $1.002-1.003$ at $25^{\circ} \mathrm{C}$, with the lighter isotope, ${ }^{6} \mathrm{Li}$, being preferentially fractionated into the resin phase. MO calculations at the HF/6-31G(d) level of theory were carried out to elucidate the lithium isotope effects experimentally obtained, assuming that the lithium species in the solution phase was the hydrated lithium ion and the lithium species in the ion exchange resin phase was the hydrated lithium ion interacting with the methyl sulfonate ion. Here, the ion exchange resin was supposed to have the sulfo group as ion exchange group, with the methyl moiety of the methyl sulfonate ion serving as the frame work of the resin. The calculations showed that the salvation number of the lithium ion was four both in the solution phase and in the ion exchange resin phase, and additional water molecules moved off to the secondary or higher salvation spheres. In Fig. 2, the rpfr value of lithium in the two phases is plotted against the hydration number. It is seen that the rpfr of the lithium ion in the resin phase is larger than that in the solution phase for the hydration number of four and smaller. For the hydration number of five and larger, the opposite is the case, which is consistent with experimental results. Thus, it 
was found that consideration on the primary salvation sphere was insufficient, leading to incorrect conclusion, and one had to take into consideration higher salvation spheres for the elucidation of the lithium isotope effects in aqueous ion exchange systems.

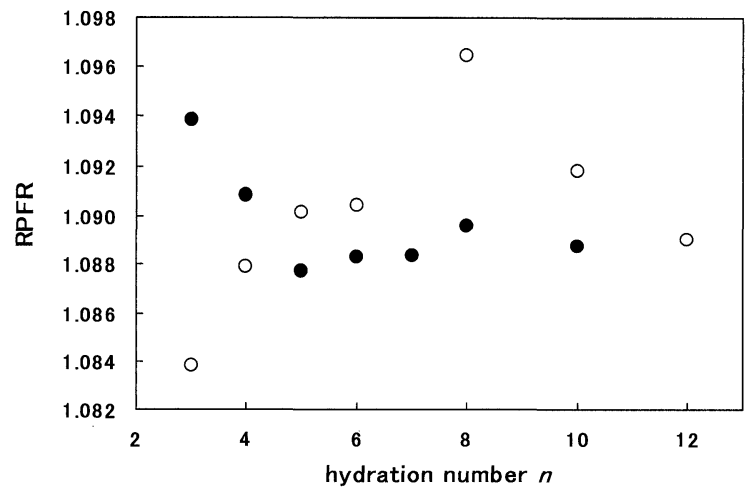

Fig.2. Plots of the reduced partition function ratios of the hydrated lithium ion $(0)$ and the hydrated lithium ion interacting with the methyl sulfonate ion $(\bullet)$ calculated at the $\mathrm{HF} / 6-31 \mathrm{G}(\mathrm{d})$ level of theory against hydration number.

\section{IV-B. Lithium isotope effect accompanying chemical insertion of lithium into graphite}

Lithium can be intercalated into graphite from, for example, 1-methoxybutane solution of lithium and naphthalene, and the lighter isotope, ${ }^{6} \mathrm{Li}$, is always preferentially fractionated into the graphite phase. ${ }^{6}$ To elucidate this experimental result, $\mathrm{MO}$ calculations at the $\mathrm{B} 3 \mathrm{LYP} / 6-311 \mathrm{G}(\mathrm{d})$ level were carried out. ${ }^{7}$ The lithium atom between the graphene layers of graphite was modeled as lithium atoms in 1:1 complexes of lithium and simple aromatic hydrocarbons with condensed benzene rings, an example of which is depicted in Fig. 3.

The largest contributions to the rpfr were from the vibrational motions in which lithium atoms vibrate perpendicularly to the condensed benzene ring planes. The lithium atom in the solution was modeled as a lithium atom interacting with a naphthalene molecule and solvated by a 1 -methoxybutane molecule in the contact ion pair manner. The rpfr values of lithium in graphite was estimated to be 1.04570 at $25^{\circ} \mathrm{C}$ and that in the 1-methoxybutane solution was 1.07126, which resulted in the equilibrium constant of 1.024 between lithium atoms in the two phases and agreed well with the experimental value of 1.023 .

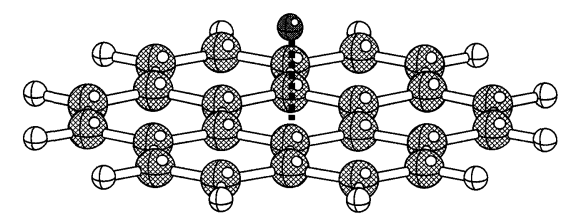

Fig.3. The structure of the $1: 1$ complex of a lithium atom and a coronene molecule considered. The intermediate sphere represents the $\mathrm{Li}$ atom and the largest and smallest ones represent $\mathrm{C}$ and $\mathrm{H}$ atoms, respectively. No significance is attached to the relative sizes of these spheres.

\section{CONCLUSION}

The estimation of rpfrs in the above two examples was never accomplished by the conventional vibrational analysis such as the one using the Wilson's $\boldsymbol{G F}$ matrix method without substantial simplification. The MO method, if the environment of the atom in concern is properly modeled, can be used to elucidate isotope effects that have experimentally observed. It would also be able to be applied to predict the magnitude of isotope effects in the systems for which experiments are not conducted or are difficult to conduct. With further developments of computational quantum chemistry and computers in terms of calculation speed and memory availability, accurate prediction of isotope effects of any element even in large and highly complicated systems may become possible in near future.

\section{REFERENCES}

${ }^{1}$ J. Bigeleisen and M.G. Mayer, J. Chem. Phys. 15, 261 (1947).

${ }^{2}$ M.J. Stern, W.A. Van Hook and M. Wolfsberg, 
J. Chem. Phys. 39, 3179 (1963).

${ }^{3}$ M.J. Frisch, et al., Gaussian 98 Rev. A.11.3, Gaussian Inc., Pittsburg, 1998.

${ }^{4}$ For instance, T. Oi, K. Kawada, M. Hosoe and H. Kakihana, Sep. Sci. Technol. 26, 1353 (1991).

${ }^{5}$ S. Yanase and T. Oi, Z. Naturforsch. 56a, 297 (2001).

${ }^{6}$ S. Hashikawa, S. Yanase and T. Oi, Z. Naturforsch. 57a, 857 (2002).

${ }^{7}$ T. Oi and S. Yanase, Z. Naturforsch. 58a, 325 (2003).

Takao Oi (大井 隆夫) was born in 1952. He graduated from Department of Applied Physics, Faculty of Science, Tokyo Institute of
Technology, in 1974, and finished his Doctor's course in Nuclear Engineering in 1979. He received a doctorate in Engineering from Tokyo Institute of Technology in 1979. From 1979 to 1984 , he worked at State University of New York at Stony Brook, USA, as a postdoctoral research associate. In 1984, he joined Department of Chemistry, Faculty of Science and Technology, Sophia University, as a research assistant, and now he is a professor of the same Department. His specialty is science and technology of isotopes including isotope separation, isotope geochemistry and elucidation of isotope effects based on molecular orbital calculations. 